\title{
Retour sur « La Mise au propre en architecture »
}

\section{Monique Eleb}

\section{OpenEdition}

\section{Journals}

Édition électronique

URL : https://journals.openedition.org/tc/5101

DOI : $10.4000 /$ tc. 5101

ISSN : 1952-420X

\section{Éditeur}

Éditions de l'EHESS

\section{Édition imprimée}

Date de publication : 30 juin 2010

Pagination : 586-588

ISSN : 0248-6016

\section{Référence électronique}

Monique Eleb, « Retour sur « La Mise au propre en architecture »», Techniques \& Culture [En ligne], 54-55 | 2010, mis en ligne le 30 juin 2013, consulté le 29 septembre 2022. URL : http:// journals.openedition.org/tc/5101; DOI : https://doi.org/10.4000/tc.5101

Ce document a été généré automatiquement le 29 septembre 2022.

Tous droits réservés 


\section{Retour sur « La Mise au propre en architecture »}

\section{Monique Eleb}

Equipement de salle de bains à l'intérieur d'une cabine - Carnival Valor (navire 2004), cliché C. Kramer 2008

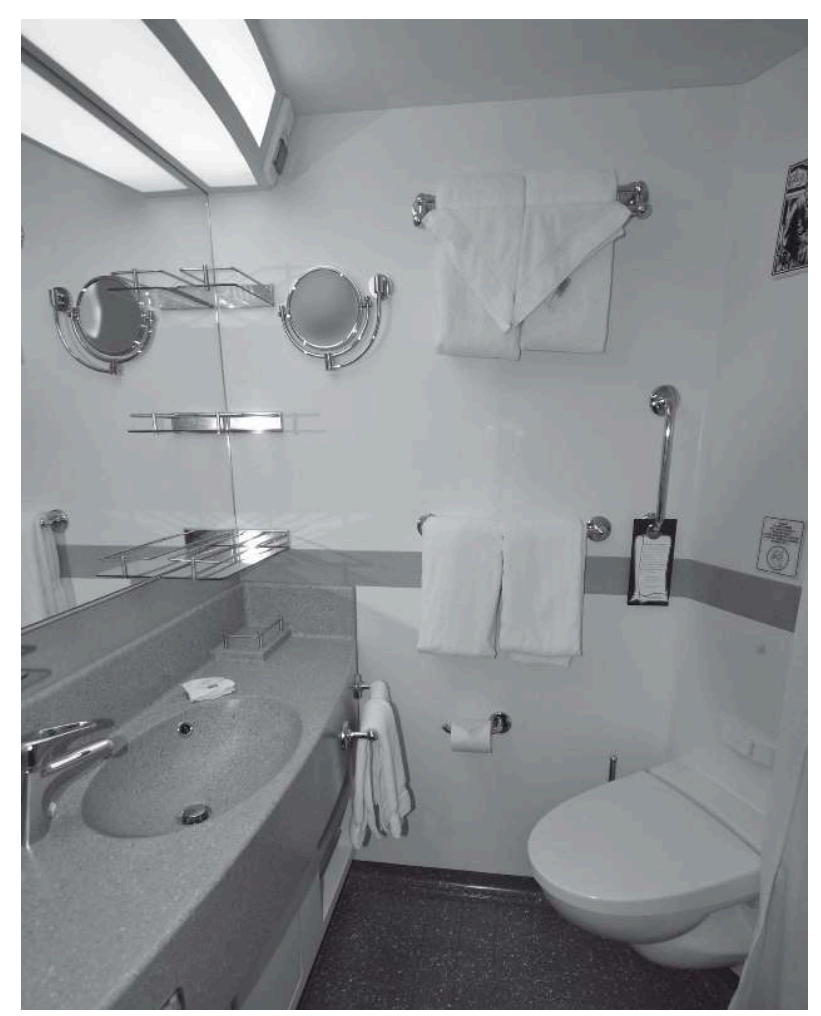

1 Nous avons échappé, au cours du $\mathrm{xx}^{\mathrm{e}}$ siècle, à bien des solutions qui nous apparaissent aujourd'hui étonnantes comme le bloc-eau des années cinquante installé dans le logement populaire, qui servait à la fois de lavoir et de douche et dans lequel il fallait se hisser en grimpant sur un tabouret, ou comme le poliban, objet du même ordre dans 
lequel on s'asseyait et qui faisait fonction de lavoir, de bain et de bidet. Ces solutions, qui ne ménageaient pas le corps, ont fait long feu et les équipements se sont unifiés, ressemblant pour la plupart aux publicités des années 1910. En fait la salle de bains a peu évolué au cours du $\mathrm{xx}^{\mathrm{e}}$ siècle, ce qui explique peut-être qu'elle soit si peu étudiée sauf par les designers et les revues de décoration qui tentent de lancer des tendances.

2 Mais à y regarder de plus près quelques transformations se sont imposées ces dernières décennies. Ainsi, par exemple, la place de la salle de bains, dans le logement, a changé. Elle se trouve, depuis les années quatre-vingt, dans le corps du logement; elle n'est plus éclairée et aérée naturellement mais par "ventilation mécanique contrôlée » (VMC), ce qui aboutit à faire commencer ses journées, quand on habite un logement récent, dans un petit réduit noir sans fenêtre. Quelques architectes résistent à cette habitude de conception et se font un devoir de donner à la salle de bains une fenêtre, mais c'est un exploit, car le linéaire de façade est coûteux, on y ouvre donc plutôt les pièces principales. De plus, l'habitude s'est établie, pour des raisons économiques une fois de plus, de construire des bâtiments épais qui conduisent à placer, en leur centre, des pièces encore appelées secondaires. Quelques architectes lui donnent la taille d'une chambre et une fenêtre. Dans l'immeuble de Jean Nouvel "Nemausus » à Nîmes, une habitante m'a dit avec fierté : "J'ai un salon de bains. » Dans quelques exemples, un muret à mi-hauteur sépare la pièce en deux parties, salle de bains et buanderie.

Depuis les années cinquante, la taille et la hauteur des équipements de la salle de bain s'étaient stabilisées, les normes de dimension s'imposant ainsi à tous et il devenait difficile de trouver un lavabo hors normes. Aujourd'hui, l'offre commence à s'élargir, les fabricants ont réduit le volume de certaines gammes d'appareils pour s'adapter à la réalité du marché et à la réduction de surface de la salle de bains. $1 \mathrm{l}$ faut noter la disparition du bidet, qui renvoyait à une très longue tradition consistant à ne pas se laver complètement mais par « morceaux ». Si le bidet a disparu en France, il continue dans certains pays (notamment en Italie) à être signe de luxe.

Cette salle de bains blanche et carrelée, aseptisée, courante aujourd'hui, et dont le but est de rendre propre et présentable, était une des solutions parmi d'autres à la fin du $x^{x}{ }^{e}$ siècle. On aurait pu retenir l'idée d'une pièce organisée pour la détente et le plaisir comme l'étaient d'ailleurs les cabinets de toilette richement meublés de la grande bourgeoisie. Aujourd'hui la visée purement hygiénique s'est pourtant éloignée tout autant que l'obéissance aveugle à l'ergonomie de la part des producteurs d'objets. La conception du corps ayant changé, le plaisir s'est introduit dans la pensée sur ces objets techniques mais on ne le perçoit pas encore dans la production courante. Les designers tentent de proposer des formes originales aux équipements qu'ils installent parfois dans la chambre ; cette idée n'est pas nouvelle. Les revues de décoration en sont pleines pourtant dans la réalité ces exemples sont rarissimes.

Autre direction montrant une évolution possible dont tous les groupes sociaux pourraient à terme bénéficier : la réflexion sur le vieillissement de la population va entraîner avec l'augmentation de la taille de la salle de bains, la multiplication des douches de plain-pied au sol en pente avec siphon central, remplaçant le bac courant en porcelaine ou en acrylique ou le "receveur de douche extra-plat». On nomme en France ce dispositif " douche à l'italienne » alors qu'on le trouve partout ailleurs dans le monde. On le voit installé chez les personnes âgées, ce qui nécessite des petits travaux, mais, de plus en plus, la demande augmente chez des personnes valides prévoyantes. 
6 Faire des salles de bains des pièces à part entière, mieux les lier aux chambres, les éclairer naturellement, est le but de certains architectes. Certains habitants expriment le désir d'un lieu où faire sa petite gymnastique personnelle, où se relaxer sur un fauteuil ou un lit de repos. Cela devient possible quand les contraintes sont mises entre parenthèses et plus aisé à réaliser dans la maison individuelle. Dans ce cas, la salle de bains renoue avec l'hypothèse, écartée au début $\mathrm{du} \mathrm{xx}^{\mathrm{e}}$ siècle, de concevoir cette pièce comme un espace de détente. On note aussi un retour de l'hydro-massage : la baignoire à remous ou à jets ne cesse de gagner du terrain en France malgré les diktats vertueux des écologistes qui préconisent la douche moins coûteuse en eau. Ce nouveau type de baignoires fait justement passer la salle de bains, de lieu d'hygiène à celui de plaisir et de délassement. Ces dispositifs et ces aménagements sont le signe que l'on commence en France à se rapprocher des techniques corporelles orientales qui associent propreté et détente.

\section{BIBLIOGRAPHIE}

Bendimérad S. \& Eleb M. 2010 « Lieux, gestes et mots du confort chez-soi » in Sabri Bendimérad \& Monique Eleb (dir.) Vu de l'intérieur. Habiter un immeuble en Île de France (1945-2010). Archibooks et Sautereau éditeur.

Csergo J., Guerrand R.-H. 1997 Le Confident des dames. Le bidet du XVIII au XX'e siècle : histoire d'une intimité. Éditions La Découverte.

Eleb-Vidal M. \& Debarre-Blanchard A. 1984 «Propreté et morale, une liaison paradoxale » in Architectures de la vie privée. Maisons et mentalités XXVI ${ }^{e}$ XIXe siècles. Bruxelles : Archives de l'Architecture Moderne, 1989, 311 p. (réimp., AAM/Paris : Hazan, 1999. Préf. de Michèle Perrot).

Eleb M. 1995 « Toilette et salle de bains. Un choix de civilisation » in Monique Eleb \& Anne Debarre (dir.), L'Invention de l'habitation moderne, Paris, 1880-1914. Architectures de la vie privée tome II. Bruxelles : Archives de l'Architecture Moderne / Paris : Hazan.

- 1994 L'Apprentissage du "chez-soi" : le Groupe des maisons ouvrières, Paris, Avenue Daumesnil, 1908. Marseille : Éditions Parenthèses, $122 \mathrm{p}$.

\section{AUTEUR}

MONIQUE ELEB

ENSAP-M, Laboratoire ACS, monique.eleb@wanadoo.fr 